Check for updates

Cite this: J. Mater. Chem. C, 2019, 7,2250

Received 24th September 2018 Accepted 4th December 2018

DOI: $10.1039 / \mathrm{c} 8 \mathrm{tc} 04818 \mathrm{e}$

rsc.li/materials-c

\section{Tailored fluorescent solvatochromic test strips for quantitative on-site detection of gasoline fuel adulteration $\dagger$}

\author{
Raúl Gotor, Jérémy Bell (D) * and Knut Rurack (D)
}

Gasoline adulteration is a frequent problem world-wide, because of the chance of quick, maximized profits. However, addition of cheaper ethanol or hydrocarbons like kerosene does not only result in economic damage but also poses problems for vehicles and the environment. To enable law enforcement forces, customers or enterprises to uncover such a fraudulent activity directly upon suspicion and without the need to organize for sampling and laboratory analysis, we developed a simple strip-based chemical test. Key to the favorable performance was the dedicated materials tailoring, which led to test strips that consisted of a cellulose support coated with silica, passivated with hexamethyldisilazane and functionalized covalently with a molecular probe. The probe fluoresces brightly across a broad solvent polarity range, enabling reliable quantitative measurements and data analysis with a conventional smartphone. The assays showed high reproducibility and accuracy, allowing not only for the detection of gasoline adulteration but also for the on-site monitoring of the quality of commercial E10 gasoline.

\section{Introduction}

Adulteration of gasoline is a recurrent problem world-wide, especially in countries without strong regulations and powerful control agencies like in various parts of South Asia, South and Central America or Africa, ${ }^{1}$ yet also in North America or Europe, ${ }^{2}$ where refined fuels do not always reach the required specifications yet are nonetheless spread onto the market. Its truly intensive and global use allows such illegal practices to seem attractive for maximizing profit. Gasoline dilution can be easily achieved with cheap and less taxed solvents of similar composition, such as mineral spirits, kerosene, rubber solvents, petrochemical naphtha, diesel, and thinner or larger amounts of alcohol, mainly ethanol. From the various surveys made by control agencies or scientists, it is apparent that the most common adulteration involves the addition of either inexpensive kerosene or ethanol. Besides unfair market competition and the influence on fuel prices, adulteration induces risks for engines like problematic starting, faster knocking, vapor lock or rubber corrosion. Moreover, depending on the

Chemical and Optical Sensing Division, Bundesanstalt für Materialforschung und prüfung (BAM), Richard-Willstätter-Str. 11, 12489 Berlin, Germany. E-mail: jeremy.bell@bam.de

$\dagger$ Electronic supplementary information (ESI) available: Materials, methods and instrumentation, procedures, photo-physical properties, test-strip preparation and analysis, and sensing of gasoline/kerosene and gasoline/ethanol mixtures. See DOI: $10.1039 / \mathrm{c} 8 \mathrm{tc} 04818 \mathrm{e}$ adulterant used, excessive toxic gas emission, e.g., $\mathrm{CO}$ and $\mathrm{NO}_{X}$, may occur. Methods to control and uncover such illegal practices have been thus developed over the years. Today, the portfolio includes $\mathrm{GC}^{3}$ and IR/Raman analysis ${ }^{4}$ as well as, according to the compilation of the ASTM (American Society for Testing and Materials), standard methods for gasoline analysis based on evaporation, density, distillation and ash content determination. ${ }^{5}$ Another common technique is to mark the original refined fuels with tracers, ${ }^{6}$ usually coloured dyes, ${ }^{7}$ which, however, is costintensive on a larger scale. All these methods also have limitations in terms of portability and in-field analysis. Thus, there is a global demand for embedded, inexpensive analytical tools to certify the quality and authenticity of gasoline. Although some approaches toward colorimetric probes for the detection of fuel adulteration have been reported recently, these either require laboratory instrumentation for measurement, are only capable of performing rough quantitative analysis or can only be used for determining adulteration with ethanol or other more polar liquids, but not with nonpolar adulterants such as kerosene. ${ }^{8}$ Unequivocal and reliable quantification with a rapid test coupled with a handheld embedded device has not been accomplished yet. With such a tool, untrained personnel could carry out surveillance campaigns or monitor directly the fuel quality at a site of suspicion. The number of samples that must be collected, transported to and analysed in the laboratory could be drastically reduced, saving cost and time. Only ambiguous or obvious adulteration cases would have to be validated in the lab, for instance, if legal actions should or need to be taken. 

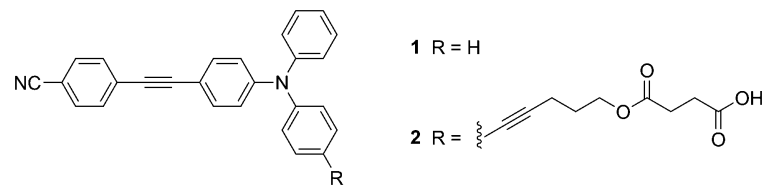

Scheme 1 Molecular structures of the fluorescent probes 1 and 2.

We report here on tailored fluorometric test strips for the determination of adulteration of gasoline with kerosene or ethanol, relying on the solvatochromic properties of highly dipolar charge transfer (CT) dyes. Molecular probe 2 with a triphenylamine moiety as an electron donor (D), phenylacetylene as a $\pi$-conjugating unit, a cyanide group as an electron acceptor (A) and a carboxylic acid-terminating linker for covalent anchoring to a support was conceived as the responsive component (Scheme 1). Key to the unique assay performance, however, was the chemical tailoring of the strips. Only when 2 was covalently grafted onto silica-coated and specifically-passivated cellulose did the analytical test strips unveil their favourable sensing behaviour. When employed in combination with a smartphone carrying a 3D-printed case, with a self-powered excitation source, reliable quantitative measurements could be realized without the need for a laboratory environment. This contribution shows how very simple analytical systems can become powerful aids in everyday analytics through dedicated materials tailoring, molecular probe design and device embedding.

\section{Results and discussion}

\section{Synthesis and photophysical properties of the fluorescent molecular dyes}

Molecular probe 1 was prepared by following a synthesis procedure previously reported by Piao et al. ${ }^{9}$ and 2 was synthesized by an adapted method, starting from dibrominated triphenylamine (Scheme 2). First, 4,4'-dibromotriphenylamine was coupled with trimethylsilyl acetylene using $\mathrm{Pd}\left(\mathrm{PPh}_{3}\right)_{2} \mathrm{Cl}_{2}, \mathrm{Cu}(\mathrm{I})$ and THF/Et ${ }_{3} \mathrm{~N}$ as a solvent mixture at $80{ }^{\circ} \mathrm{C}$ in a Sonogashira coupling reaction. The mono-functionalized product 3 was separated from the statistical distribution mixture of products and was deprotected with potassium carbonate in a $\mathrm{MeOH} / \mathrm{CH}_{2} \mathrm{Cl}_{2}$ mixture. Then, 3

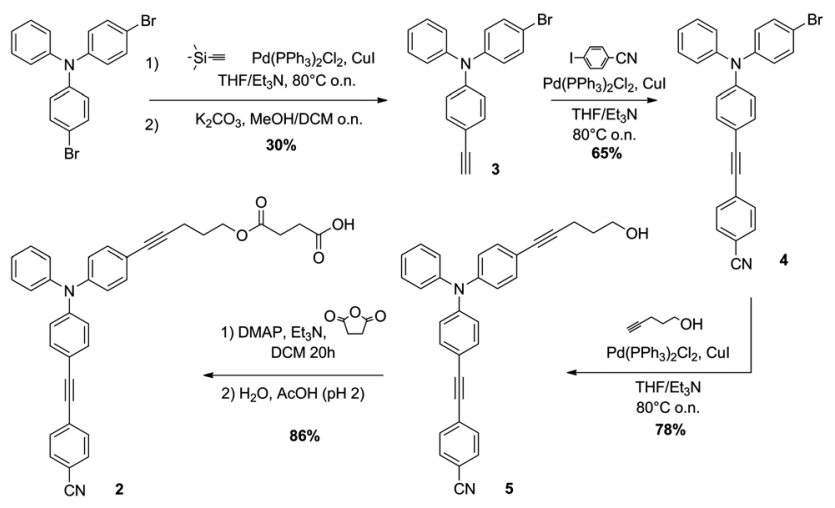

Scheme 2 Synthesis of the molecular probe 2. was coupled with 4-iodobenzonitrile in a Sonogashira coupling reaction to produce 4 in $65 \%$ yield. This compound was again subjected to a Sonogashira coupling reaction, this time using 4-pentyn-1-ol to obtain 5, which was subjected to an esterification reaction with succinic anhydride and DMAP to form 2 with an overall yield of 13\%. Molecular probes 1 and 2 exhibited typical photophysical properties of $\pi$-extended charge transfer (CT) dyes with absorption in the UV-blue range and emission with high fluorescence quantum yields in the blue-green range of the spectrum.

The virtually identical properties of the two dyes indicated the negligible influence of the linker substituent for 2 (Table S1 and Fig. 1, Fig. S2, ESI $\dagger$ ). Both dyes also presented a large Stokes shift from 40 to $200 \mathrm{~nm}$ depending on the solvent polarity. Consistent with a pronounced increase in the dipole moment after charge-transfer excitation of these types of dyes, the increasing Stokes shift is primarily related to the strong red shift of the emission band from 401 to $543 \mathrm{~nm}$. Using the LippertMataga formalism (eqn (1), (2) and Fig. 1), ${ }^{10}$ the slope values proportional to the cavity size and the polarity of the excited states $\left(\Delta \mu^{2} / a^{3}\right)$ can be calculated.

$$
\begin{gathered}
\nu_{\mathrm{a}}-\nu_{\mathrm{b}}=\frac{2}{h c a^{3}}\left(\mu_{\mathrm{e}}-\mu_{\mathrm{g}}\right)^{2} \Delta f+C \\
\Delta f=\frac{\varepsilon-1}{2 \varepsilon+1}-\frac{n^{2}-1}{2 n^{2}+1}
\end{gathered}
$$

Compared to CT dyes that are similar in shape and size (i.e., D-Asubstituted acetylene-bridged triarylamines), ${ }^{11}$ the title dyes show distinctly larger values, indicating a considerable dipole moment of the excited state. This pronounced solvatochromic behaviour
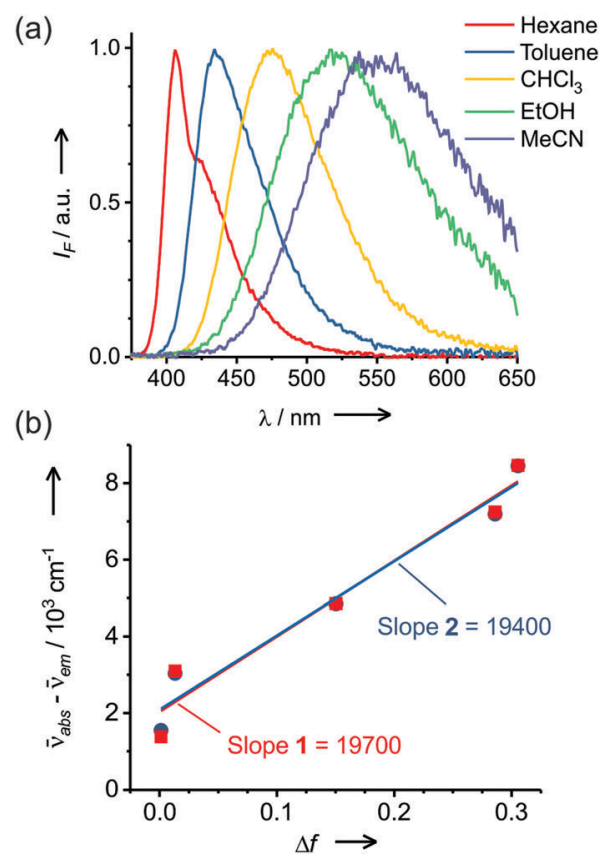

Fig. 1 (a) Normalized emission spectra of $\mathbf{2}$ in various solvents and (b) Lippert-Mataga correlations for CT dyes 1 and 2 . The error in the Stokes shift is $10 \mathrm{~cm}^{-1}\left(c_{1}=c_{2}=4 \mu \mathrm{M}, \lambda_{\mathrm{exc}}=340 \mathrm{~nm}\right)$. 
suggests the availability of a broad dynamic measurement range when employing the dyes for the application in question (Fig. S5c, ESI $\dagger$ ). Interestingly, despite the fluorescence band shifting by more than $140 \mathrm{~nm}$, the influence of the solvents on the probes' fluorescence quantum yield $\left(\Phi_{\mathrm{f}}\right)$ is minor. While for this family of dyes, polar solvents can potentially promote strong intramolecular CT and non-radiative deactivation pathways, here, only in acetonitrile is the $\Phi_{\mathrm{f}}$ of $\mathbf{1}$ and 2 somewhat lower at 58\% and $37 \%$. Probe 1 exhibited a resolved emission band at $403 \mathrm{~nm}$ in decane, which was first used to simulate complex fuel mixtures without potentially interfering additives, and a broad emission band at $511 \mathrm{~nm}$ in ethanol (Fig. S5a, ESI $\dagger$ ).

Since gasoline can be auto-fluorescent because of the possible presence of fluorescent polycyclic aromatic hydrocarbons or even marker dyes, a previously reported embedded protocol using a stainless steel in-line filter holder with active carbon paper filters was applied here as well. ${ }^{12}$ In gasoline E5, probe 2 showed an emission band centred at $435 \mathrm{~nm}$, which underwent a bathochromic shift of $86 \mathrm{~nm}\left(\lambda_{\mathrm{em}}=521 \mathrm{~nm}\right)$ upon increasing the proportion of ethanol, showing no disturbance from the fuel matrix (Fig. 2a, b and Fig. S5b, ESI $\dagger$ ).

Favourable hydrogen bonding interactions between ethanol and the polar dye led to a higher micropolarity, i.e., the polarity of the solvent shell surrounding the probe is higher compared to the overall polarity, ${ }^{13}$ especially at a low ethanol content. Because of these microsolvation phenomena, a linear shift of the emission band for ethanol proportions between 0 and 10 vol\% was observed, followed by a non-linear shift for higher proportions of up to $100 \mathrm{vol} \% \mathrm{EtOH}$. This behaviour allows the probes to be used in two assays, i.e., for detecting gasoline dilution with significant amounts of ethanol and for more accurate monitoring of gasoline E5 as directly received from the refinery. In contrast, and in agreement with a decrease of the mixture's polarity, increasing proportions of kerosene induced a hypsochromic shift of up to $24 \mathrm{~nm}\left(\lambda_{\mathrm{em}}=411 \mathrm{~nm}\right)$. This shift appeared to be linear over the entire range from 0 to 100 vol\% kerosene in the mixture. Furthermore, simple procedures for recording the response of the probes are greatly facilitated because of the similar $\Phi_{\mathrm{f}}$ values of $c a$. $80 \%$ for 1 and $60-70 \%$ for 2 in gasoline E5, ethanol and kerosene. Based on error propagation including repetition and analysis errors, ${ }^{14}$ a limit of detection $(3 \sigma)$ of 0.5 and 5.4 vol\% and a limit of quantification $(5 \sigma)$ of 1.8 and $18.0 \mathrm{vol} \%$, respectively, were calculated for ethanol and kerosene adulteration using polarity probe 2 . These values perfectly match the usual proportion of ethanol or kerosene found in adulterated gasoline E5, as less than 10 vol\% has no economic benefit while more than 30 vol\% starts to be detectable without instrumentation. ${ }^{15}$

\section{Dye functionalisation of cellulose and glass fibre paper}

These promising results led us to incorporate the molecular probe onto a solid support to facilitate in-the-field assays. As expected, simple impregnation of cellulose filter paper (Whatman 1) with probe 1 by way of a $1 \mathrm{mM}$ solution in toluene did not yield the desired performance. The ensemble was unstable and $\mathbf{1}$ diffused out of the strip when in contact with liquids, especially

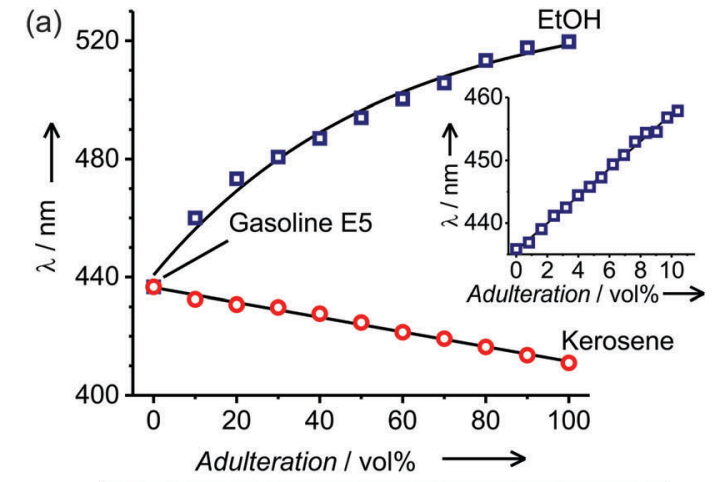

(b)

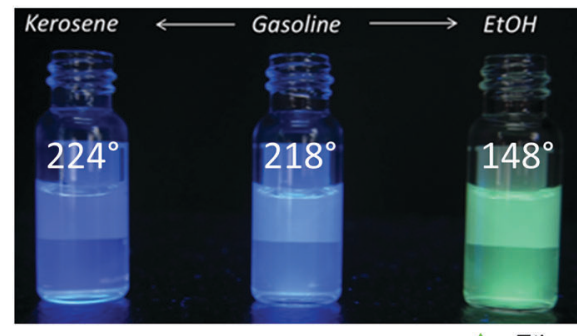

(c)

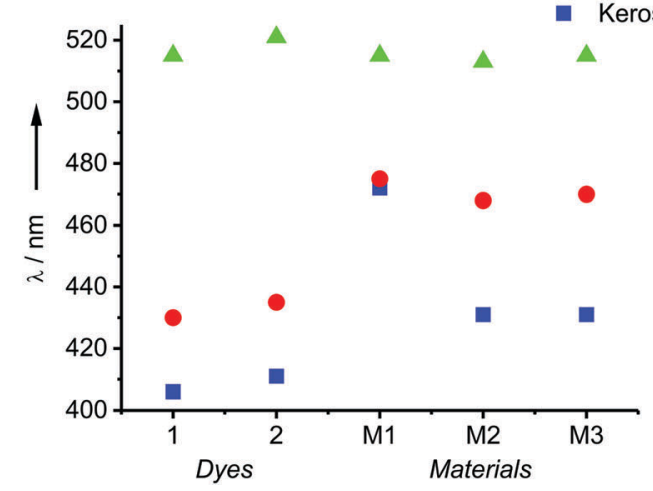

Fig. 2 (a) Emission maxima of $\mathbf{2}$ in gasoline E5/ethanol and gasoline E5/kerosene mixtures and inset with linear plot for low amounts of ethanol. (b) Photograph of solutions of $\mathbf{2}$ in kerosene, gasoline $\mathrm{E} 5$ and ethanol under a UV lamp with hue values indicated $\left(c_{2}=4 \mu \mathrm{M}, \lambda_{\mathrm{exc}}=340 \mathrm{~nm}\right)$. (c) Dynamic ranges of $\mathbf{1}$ and $\mathbf{2}$ in solution and strips M1, M2 and $\mathbf{M} \mathbf{3}$ visualized by the extreme emission maxima in kerosene (blue squares) and ethanol (green triangles); unadulterated gasoline E5 (red circles) shown as a reference.

when samples contained higher proportions of ethanol. Thus, 2 was chemically grafted onto previously aminated test strips by means of DCC coupling, yielding stable test strips. Because strong polarity variations can be expected with the type of samples being targeted, which would potentially lead to inhomogeneous swelling behaviour or diffusion processes, we opted for immobilization of 2 on standard filter paper (test strips M1, Fig. 3a) and refrained from using, for instance, a polymer matrix. In addition, we also anchored 2 to glass fibre paper (test strips $\mathbf{M} 2^{\prime}$, Fig. 3a) to screen for the optimal support. As shown in Fig. 2c, this approach yielded an acceptable performance for the detection of ethanol in gasoline, and the difference in the emission maxima of strip M1 after dipping into gasoline E5 and ethanol amounted to $\Delta \lambda_{\text {EtOH-GE5 }}=$ $33 \mathrm{~nm}$. Moreover, spectral analysis of various gasoline E5/ethanol mixtures showed a linear dynamic range from 0 to $20 \mathrm{vol} \%$ with an 
(a)
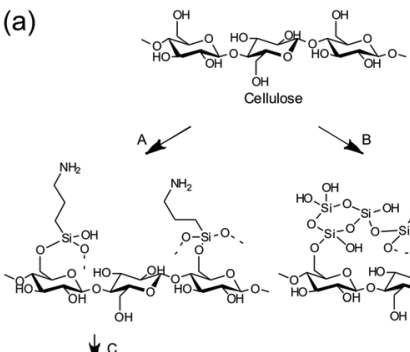

${ }^{B}$

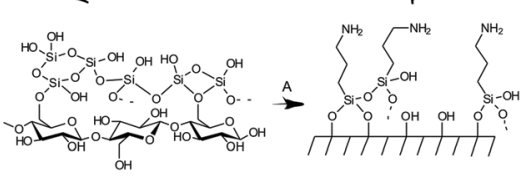
$\downarrow c$

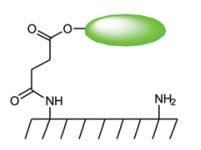

m1
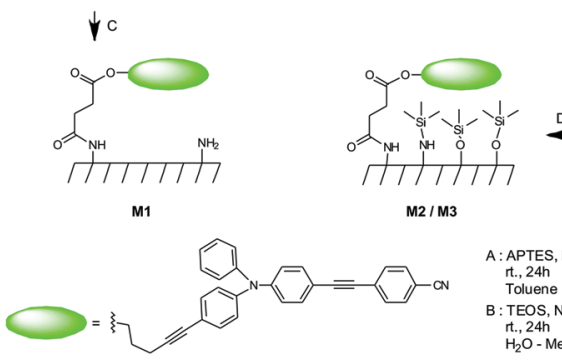

M2 / M3

(b)

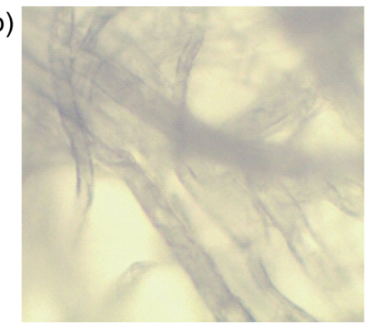

(d)

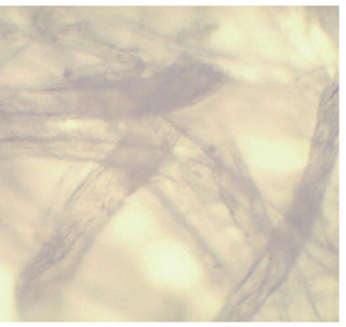

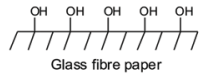

$r^{c}$

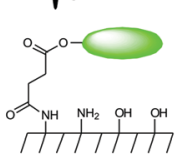

M2' / M3'

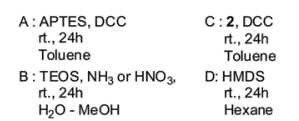

(c)

(e)

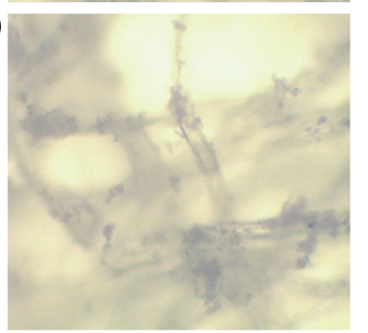

Fig. 3 (a) Scheme representing the preparations of the different test strips M1, M2', M2, M3', and M3. Microscopy photographs of cellulose without coating (b) and silica-coated cellulose strip obtained (c) under acidic conditions $\left(\mathrm{HNO}_{3}, \# 4\right)$, (d) with 0.7 equivalents of ammonia vs. TEOS (\#6) and (e) 0.2 equivalents of ammonia vs. TEOS (\#10).

average error of \pm 5 vol\%, which is promising regarding the polar sensing capability. For comparison, the respective range was only $0-10$ vol\% in solution, most likely because of the higher $\Delta \lambda_{\text {EtOH-GE5 }}=86 \mathrm{~nm}$. However, the discrimination capability of M1 on the nonpolar side was negligible with the emission maximum in kerosene being only slightly blue-shifted with $\Delta \lambda_{\mathrm{K}-\mathrm{GE} 5}=-3 \mathrm{~nm}$. Compared to 2 in solution, this strongly reduced measurement range indicates the negative influence of the polar aminated cellulose matrix. Assays done with $\mathbf{M} 2^{\prime}$ strips showed similar results with $\Delta \lambda_{\mathrm{K}-\mathrm{GE} 5}=-10 \mathrm{~nm}$.

To improve the dynamic working range of the strips, especially for nonpolar mixtures, and make the assay viable for both of the extreme adulteration cases (extreme in terms of the polarity difference of the compounds involved), $\mathbf{M} 2^{\prime}$ was passivated with hexamethyldisilazane (HMDS), yielding M2 (Fig. 3a). ${ }^{16}$ The success of this strategy can be seen in Fig. 2c. The passivated M2 now showed a much broader dynamic range with $\Delta \lambda_{\text {EtOH-GE5 }}=45 \mathrm{~nm}$

and $\Delta \lambda_{\mathrm{K}-\mathrm{GE} 5}=-37 \mathrm{~nm}$, respectively, allowing the detection of both kerosene and ethanol mixtures with an error of \pm 8 vol\%. To combine the glass fibre strips' chemical flexibility with the paper strips' robustness-glass fibre paper is rather fragile and not wellsuited for use by untrained personnel-M3 strips were prepared by first coating silica onto the filter paper, ${ }^{17}$ grafting the dye and then passivating it with HMDS (Table S2, ESI $\dagger$ ). Coating of the cellulose fibres was attempted using TEOS (tetraethoxysilane) in a water/ ethanol mixture with either ammonia or nitric acid as the catalyst. From the gain in weight and infrared analysis (Fig. S3, ESI $\dagger$ ), negligible coating with silica and even a loss of cellulose were observed under acidic conditions (\#4). In contrast, basic catalysis yielded gains of 23.8 and $26.6 \mathrm{wt} \%$ for 0.7 and 0.2 equivalents of ammonia vs. TEOS, respectively (entries \#6 and \#10, Table S2, ESI $\dagger$ ).

The strain of the cellulose strips and the fibre structure remained largely unaltered (2.7-4.5\%) for all the conditions tested. Photographs of the cellulose strips (Fig. 3b-e) showed that the silica coating was homogeneous only when 0.7 equivalents of ammonia were used (\#6), and 0.2 equivalents results in "silica blossoms" growing from the fibres (\#10). In accordance with the higher specific surface available, grafting led to $\mathbf{M} 3_{\# \mathbf{1 0}}$ $\left(20.5 \mu \mathrm{mol} \mathrm{g}^{-1}\right)$ being functionalized with higher amounts of probe 2 than $\mathbf{M 3}_{\# 6}\left(9.4 \mu \mathrm{mol} \mathrm{g}{ }^{-1}\right)$. Under UV excitation, the homogeneous strips $\mathbf{M} 3_{\# \mathbf{6}}$ showed less fluorescence than the blossomed strips $\mathbf{M} 3_{\# \mathbf{1 0}}$ (Fig. S4, ESI $\dagger$ ). However, assays showed a broader Hue variation for $\mathbf{M} 3_{\# 6}\left(\Delta \mathrm{Hue}_{\mathrm{EtOH}-\mathrm{K}}=45^{\circ}\right)$ compared with $\mathbf{M 3}_{\# \mathbf{1 0}}\left(\Delta \mathrm{Hue}_{\mathrm{EtOH}-\mathrm{K}}=35^{\circ}\right)$ and $\mathbf{M} 3_{\# \mathbf{6}}$ was therefore selected as M3.

\section{Application to gasoline adulteration sensing}

The response kinetics and especially the assay times are important features of simple fluorescence methods (Fig. S7, ESI $\dagger$ ). Determined by the time that thorough wetting of the strip requires and the volatility of the analytes, the most reproducible results were obtained for the measurement of the emission $\mathrm{ca} .60 \mathrm{~s}$ after dropping of the fuel on the test strip. After the first minute, wetting of the strip is completed. This stable signal is maintained for $c a .100 \mathrm{~s}$, before evaporation of volatile components resulted in a colour/fluorescence change. When the strip was directly dipped into a fuel for a couple of seconds, the measurement could take place in a relatively large time window of up to $100 \mathrm{~s}$, before the fuels started to evaporate. + Only pure ethanol exhibited an unstable signal due to its high volatility. Being interested in the robustness of $\mathbf{M 1}$ and $\mathbf{M 3}$, single test strips were used for successive measurements alternatively by wetting in gasoline E5, kerosene and ethanol with a washing (by acetone) and drying step (in air for $10 \mathrm{~s}$ ) in between. For all the materials, no leaking of the dye was observed, and the optical responses remained stable over the measurements (Fig. S8, ESI $\dagger$ ). Although the strips are meant for disposable use, this feature contributes to their ruggedness.

Taking advantage of the capability of conventional mobile communication devices to act as potent detectors for simple

$\ddagger$ Usage of a smartphone for measurement and data analysis circumvents timing problems by inserting the strip directly after dipping, starting the measurement and leaving the strip for ca. $5 \mathrm{~min}$ in the case. The software then determines the plateau from a sequence of photographs and extracts the relevant data. 
(a)

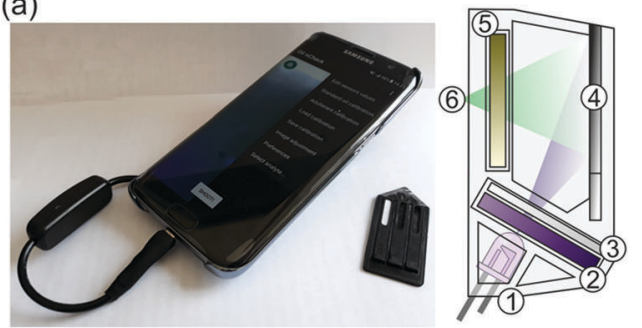

(b)

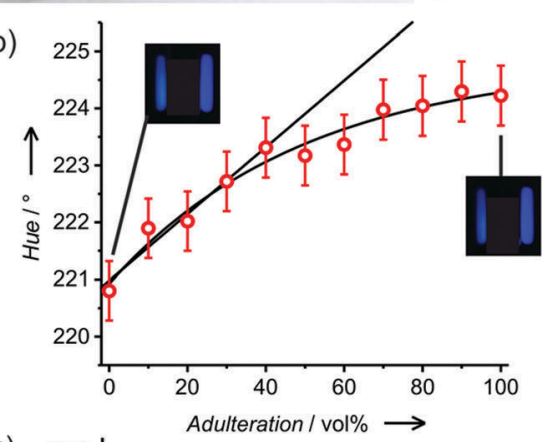

(c)

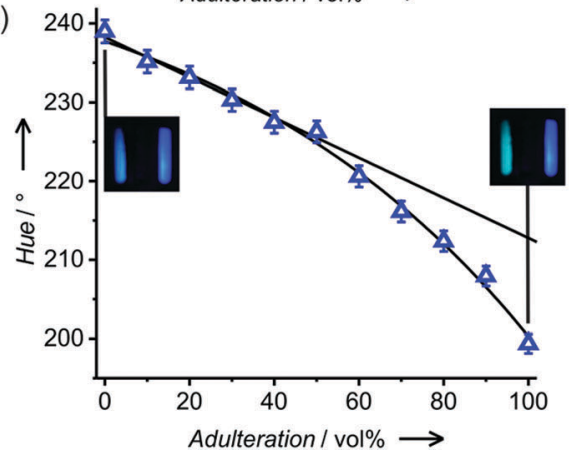

Fig. 4 (a) Picture and schematic representation of the smartphone case integrating: (1) $380 \mathrm{~nm}$ LED, (2) excitation filter, (3) diffuser, (4) test strip slot and fitting, (5) emission filter and (6) position of the smart-phone CMOS. ( $b$ and $c$ ) Respective hue values measured from M3 test-strips when dipped in gasoline E5/kerosene and gasoline E5/ethanol mixtures. Respective dynamic ranges for the determination of the kerosene and ethanol content in gasoline E5 span $19-40 \%$ and $4-50 \%$, respectively.

chemical assays, a smartphone-based measurement system analysing the fluorescence of $\mathbf{M} 3$ test strips was designed (Fig. 4a and Fig. S6, ESI $\dagger$ ). Measurement systems relying on such a combination are ideally suited for on-site testing by unskilled personnel, allowing the immediate transfer of the obtained data and a respective response action. When measuring fluorescence, smartphone-based systems require an additional excitation source and a controlled dark chamber to yield accurate and reproducible results.§ We here used a 3D printed case designed for a conventional Samsung Galaxy S7 Edge phone. The case is a black chamber $(20 \times 30 \times 40 \mathrm{~mm})$, including a UV-LED $(380 \mathrm{~nm})$ driven by a DC current of $20 \mathrm{~mA}$ drawn from

$\S$ Measurements are reproducible for single phone/case combinations. Changing the type of phone or device requires adaption of the case, optimization of the parameters and/or (only) a new calibration. The type or generation of device as such does not determine the signal quality. Important are the detector size, access to RAW images, and a short focal distance to collect as much emission from the strip as possible and therefore the most possible signal.

the phone's battery via a USB on-the-go (OTG) connection for excitation. The excitation light was filtered through a band pass filter (240-395 nm, FGUV5, Thorlabs) and diffused to homogeneously illuminate the strips at an angle of $60^{\circ}$. A low background signal around $400 \mathrm{~nm}$ representing approximately $1 \%$ of the total signal corresponds mainly to some residual excitation light passing through the overlapping regions of the filter sets. No perturbing autofluorescence from the support materials upon excitation at $380 \mathrm{~nm}$ or scattering from the fibre matrices was observed (Fig. S10, ESI $\dagger$ ). Besides the actual test strip, the holder accommodates a paper strip coated with styrene as a reference. Internal referencing has recently been shown to improve the results of comparable simple tests significantly. ${ }^{18}$ Fluorescence was measured through a long pass filter (400 nm, FGL400, Thorlabs) with the phone's CMOS back camera.

Having in mind untrained personnel as potential users of these tests, a smartphone application (or "App") was programmed, integrating calibration management, data storage and control functions for specific samples. The system can be calibrated following a three-point calibration procedure with reference solutions (gasoline, ethanol or kerosene). If known, the use of parent non-adulterated gasoline or parent adulterant, especially kerosene, for calibration can increase the measurement accuracy. The calibration parameters were stored in an internal file and could be used immediately as well as in later assays. The software averaged the RGB values inside a selected area of the captured picture corresponding to the area where the strips were placed, and they were then converted to hue values for numerical analysis using the Preucil equation ${ }^{19}$ (eqn (3)).

$$
h_{\mathrm{rgb}}=a \tan 2(\sqrt{3}(G-B), 2 R-G-B)
$$

The hue value $\left(h_{\mathrm{rgb}}\right)$ represents the main property of a colour, which is here directly related to the dominant wavelength of M3 fluorescence emission. Converting the measured RGB signals into hue values appeared to be more precise and less prone to error while intrinsically correcting unexpected light intensity variation like fluctuation of the LED, unequal coating of a strip, or daylight pollution (Fig. S9, ESI $\dagger$ ). As described in a previous publication ${ }^{12}$ the measured hue values were corrected afterwards, first using the stored calibrations for pure gasoline, kerosene or ethanol grade. Secondly, a second strip coated with a reference dye in a defined concentration was placed beside the sample strip, affording a constant fluorescence emission. The hue value of this reference was then coupled to the correction factors, accounting for exposure fluctuations.

Two different ISO values had to be used for kerosene or ethanol mixtures to obtain the maximal hue variation (Table S3, ESI $\dagger$ ) after dipping (or spotting) the test strip into a gasoline E5 sample. In the case of an unknown mixture, the first picture using ISO 64 would indicate a potential adulteration with ethanol, whereas, the second picture with ISO 1600 would indicate adulteration with kerosene. Adulteration of gasoline E5 with both adulterants at the same time leads to results similar to ethanol adulteration (Table S4, ESI $\dagger$ ). System operation, image processing and data analysis were carried out using an Android 
Table 1 Analysis of gasoline E10 samples from Berlin, Germany, using M3 test strips combined with the smartphone embedded system and a commercial in-the-field test with and without image analysis (Briggs \& Stratton, Gasohol Tester, Ref. 795161)

\begin{tabular}{|c|c|c|c|c|}
\hline \multirow[b]{2}{*}{ Samples } & \multirow[b]{2}{*}{ Hue $/^{\circ}$} & \multirow[b]{2}{*}{ Total EtOH/vol\% } & \multicolumn{2}{|c|}{ Commercial test/vol\% } \\
\hline & & & Eye-reading & Computer-reading \\
\hline A & $236.5 \pm 0.6$ & $12.2 \pm 0.9$ & $9 \pm 5$ & $8.5 \pm 1.5$ \\
\hline B & $236.9 \pm 2.0$ & $10.2 \pm 3.3$ & - & - \\
\hline $\mathrm{C}$ & $237.1 \pm 0.4$ & $9.7 \pm 0.7$ & - & - \\
\hline
\end{tabular}

custom application. For both adulterants, the smartphonebased sensor showed a linear response up to $40 \mathrm{vol} \%$ with limits of detection of $19 \pm 6 \mathrm{vol} \%$ and $4 \pm 1 \mathrm{vol} \%$ for kerosene and ethanol, respectively (Fig. $4 \mathrm{~b}$ and c). To validate the method, analysis of gasoline E10 samples from Berlin petrol stations was carried out (Table 1).

The only step before dipping $\mathbf{M} 3$ strips into the samples and analysing them with the smartphone was filtering them through active charcoal, as described above. As gasoline E5 was used as a reference, the ethanol concentration calculated with the calibration curve yields the amount of adulterant plus the amount of alcohol ( $5 \mathrm{vol} \%$ ) that is already present in the E5 reference. Finally, the results showed that samples B and C were within the regulatory limits with an alcohol proportion of approximately $10 \mathrm{vol} \%$, but that sample A slightly exceeded this limit. For comparison, sample A was also analysed with the only commercially available rapid test, utilizing phase separation upon water addition to the fuel in a test tube and visual inspection. The results obtained therewith agree fairly well with those obtained with our method, yet such a test has a low sensitivity and the uncertainty of measurement is distinctly larger. Analysing the images of the test tube with a computer somewhat improves the test's performance, yet would not provide any advantage compared to the test developed by us here. These results stress the potential of the embedded system for uncovering not only adulteration but also for monitoring the quality of commercial gasoline/ethanol mixtures.

\section{Conclusions}

A simple and powerful analytical tool was developed based on suitably tailored cellulose paper test strips. Silanization, amination and passivation were the three key steps to qualify the cellulose strip to act as a powerful support for a covalently immobilized charge transfer-type molecular polarity probe. This approach afforded test strips with exceptional performance for the on-site determination of gasoline adulteration. Analysis of a very broad polarity range from 0.0099-0.654 relative polarity was possible through recording of the indicator dye's bright and colourful fluorescence with a mobile phone's camera. Smartphone embedding yielded a simple device for gasoline analysis, allowing the determination of adulteration of gasoline with kerosene or ethanol with an error of 1-6 vol\%,

T Relative polarity of hexane, approximating the relative polarity of kerosene. Values obtained from ref. 13. perfectly suitable in terms of sensitivity, dynamic range and robustness. The latter enables straightforward rapid testing on-site by untrained personnel. These simple tests could potentially be used against fake oil reselling or in an environmental context by rendering pollution management more straightforward. Additionally, M3 is an efficient fluorescent purity indicator either for lighter alkanes such as kerosene or for polar solvents like ethanol with respective limits of detection of 5.4 and 0.5 vol\%. The perfectly suitable performance of the method for ethanol adulteration would also allow for the monitoring of refined gasoline even in Europe, as shown with the example of gasoline E10 samples collected from petrol stations. This contribution has shown the potential that the chemical tailoring of the materials properties of simple supports such as paper harbours for the future development of everyday analytical tools.

\section{Conflicts of interest}

There are no conflicts to declare.

\section{Acknowledgements}

We thank Maya Friedrich (Hufeland Schule, Berlin) for help with test strip preparation and Anka Kohl (BAM Div. 1.4) for IR analysis. Funding of the TF-Project OIL by BAM' Focus Area Analytical Sciences is gratefully acknowledged.

\section{Notes and references}

1 R. Tharby, Catching Gasoline and Diesel Adulteration, World Bank, Washington DC, 2002; A. de Souza Lima, C. S. de Assis, E. Raldenes and J. P. M. L. Pereira,O Programa de Monitoramento da Qualidade dos Combustiveis - PMQC, Agência Nacional do Petróleo, Rio de Janeiro, 2012.

2 Fuel Quality in the EU in 2016, European Environment Agency, Luxembourg, 2018.

3 A. Majhi, V. S. Kukerti, Y. K. Sharma, R. Khanna and A. Datta, Pet. Sci. Technol., 2012, 30, 271; L. S. Moreira, L. A. d'Avila and D. A. Azevedo, Chromatographia, 2003, 58, 501.

4 M. A. Al-Ghouti, Y. S. Al-Degs and M. Amer, Talanta, 2008, 76, 1105; L. S. G. Teixeira, F. S. Oliveira, H. C. dos Santos, P. W. L. Cordeiro and S. Q. Almeida, Fuel, 2008, 87, 346; K. M. Tan, I. Barman, N. C. Dingari, G. P. Singh, T. F. Chia and W. L. Tok, Anal. Chem., 2013, 85, 1846.

5 ASTM D381, ASTM D4052, ASTM D86 and ASTM D482, https://www.astm.org.

6 A. Glover, N. Aziz, J. Pillmoor, D. W. J. McCallien and V. B. Croud, Fuel, 2011, 90, 2142; G. T. Tanaka, F. D. Ferreira, C. E. F. da Silva, D. L. Flumignan and J. E. de Oliveira, J. Chemom., 2011, 25, 487.

7 J. Czarnocka, U. Zborowska and A. Kęsik, Chemik, 2011, 3, 200.

8 M. El Bakkari, R. Luguya, R. Correa da Costa and J.-M. Vincent, New J. Chem., 2008, 32, 193; A. Sedighi, S. Qiu, M. C. K. Wong and P. C. H. Li, ACS Appl. Mater. Interfaces, 
2015, 7, 28387; K. Kumar and A. K. Mishra, Talanta, 2012, 100, 414; J. Lee, S. Balakrishnan, J. Cho, S.-H. Jeon and J.-M. Kim, J. Mater. Chem., 2011, 21, 2648.

9 M. J. Piao, K. Chajara, S. J. Yoon, H. M. Kim, S.-J. Jeon, T.-H. Kim, K. Song, I. Asselberghs, A. Persoons, K. Clays and B. R. Cho, J. Mater. Chem., 2006, 16, 2273.

10 E. Lippert, Z. Naturforsch., A: Phys. Sci., 1955, 10, 541; N. Mataga, Y. Kaifu and M. Koizumi, Bull. Chem. Soc. Jpn., 1955, 28, 690.

11 V. Alain-Rizzo, D. Drouin-Kucma, C. Rouxel, I. Samb, J. Bell, P. Y. Toullec, V. Michelet, I. Leray and M. Blanchard-Desce, Chem. - Asian J., 2011, 6, 1080; D. Cvejn, E. Michail, K. Seintis, M. Klikar, O. Pytela, T. Mikysek, N. Almonasy, M. Ludwig, V. Giannetas, M. Fakis and F. Bures, RSC Adv., 2016, 6, 12819; T.-C. Lin, G. S. He, P. N. Prasad and L.-S. Tan, J. Mater. Chem., 2004, 14, 982.

12 R. Gotor, C. Tiebe, J. Schlischka, J. Bell and K. Rurack, Energy Fuels, 2017, 31, 11594.
13 C. Reichardt and T. Welton, Solvents and Solvent Effects in Organic Chemistry, Wiley-VCH, Weinheim, 2010, 359.

14 G. L. Long and J. D. Winefordner, Anal. Chem., 1983, 55, 712A-724A; K. Rurack and M. Spieles, Anal. Chem., 2011, 83, 1232-1242.

15 L. S. M. Wiedemann, L. A. d'Avila and D. A. Azevedo, Fuel, 2005, 84, 467.

16 A. B. Descalzo, M. D. Marcos, C. Monte, R. Martinez-Manez and K. Rurack, J. Mater. Chem., 2007, 17, 4716.

17 S. Sequeira, D. V. Evtuguin, I. Portugal and A. P. Esculcas, Mater. Sci. Eng., C, 2007, 27, 172; J. Cai, S. Liu, J. Feng, S. Kimura, M. Wada, S. Kuga and L. Zhang, Angew. Chem., Int. Ed., 2012, 51, 2076.

18 P. Ashokkumar, H. Weißhoff, W. Kraus and K. Rurack, Angew. Chem., Int. Ed., 2014, 53, 2225.

19 F. Preucil, Proc. - TAGA, 1953, 102. 